\title{
La complejidad de tarea de las ocupaciones ${ }^{1}$
}

\author{
JAIME RIVIÈRE \\ Universidad de Salamanca \\ riviere@usal.es
}

Recibido: 06.11.2008

Aceptado: 26.05.2009

Las ocupaciones se suelen definir como el paquete de tareas que conforman el ámbito de una determinada actividad laboral. Las diferencias entre ellas, en principio, son básicamente de carácter cualitativo. Su estudio se ha realizado o bien por medio de las propias clasificaciones de ocupaciones, siguiendo una estrategia extensiva, o por medio de estudios en profundidad de difícil extrapolación, siguiendo una estrategia intensiva (Abbot, 1993; Zhou, 1992). La mayor parte de los argumentos sobre la relevancia de las ocupaciones, sin embargo, ha hecho tradicionalmente referencia a determinados rasgos o características de éstas (Desrosieres y Thevenot, 1990; Wolf, 1997 ). Un ejemplo contundente es el de la distinción entre ocupaciones manuales y no manuales: no es, por si misma, una distinción $\mathrm{de} \mathrm{grado}^{2}$, sino una delimitación cualitativa que hace referencia a diferencias sustanciales en relación con el tipo de trabajo y, por supuesto, también a sus consecuencias sociales (Coxon y Jones, 1979; O'Reilly y Rose, 1998). El análisis de las ocupaciones por medio de las clasificaciones al uso constituye una estrategia natural para enfrentarse con este tipo de diferencias internas en las ocupaciones, pero no captura en todos los casos las sutilezas de las diferencias ocupacionales, y deja a las inevitables arbitrariedades de las decisiones clasificatorias la capacidad para articular y manipular la información relevante (Carabaña y Gomez Bueno, 1994 y 1996; Thevenot, 1983).

Una estrategia alternativa consiste en tratar a las ocupaciones según las características de su contenido en tareas. De este modo, las propiedades de los em-

\footnotetext{
${ }^{1}$ Debo agradecer a dos evaluadores anónimos el esfuerzo por procurar que este artículo fuera presentable. Sus propuestas, que he intentado seguir en la medida de mis posibilidades, han sido fundamentales para aclarar los argumentos y señalar los resultados más relevantes.

2 No es una distinción de grado por sí misma, dado que no hay una propiedad ordenable e intrínseca con respecto a la cual se establezca la distinción, aunque pueda ordenarse con respecto a sus consecuencias sociales o, como hacemos en este estudio, con respecto a propiedades internas de las ocupaciones que contiene.
}

EMPIRIA. Revista de Metodología de Ciencias Sociales. N. ${ }^{\circ}$ 17, enero-junio, 2009, pp. 91-121. ISSN: 1139-5737 
pleos se definen una por una y por separado según aspectos generales, y no a partir de un título ocupacional que reúna todo su contenido. En este caso, la estrategia no tiene como objetivo la clasificación o tipificación, sino la exploración de las consecuencias sociales de las características de las ocupaciones. A este modo de trabajar con las ocupaciones se le ha denominado analítico, por oposición a los procedimientos sintéticos o tipológicos de estudio ocupacional basado en las clasificaciones oficiales (Para una discusión general del uso en ciencia de ambos tipos de estrategia, ver Wirth, 1993) ${ }^{3}$. A pesar de sus ventajas, las aproximaciones analíticas al estudio de las ocupaciones no abundan en la literatura. El intento más exitoso es el realizado por Garrido (1991), en el que se realizaba una asignación de características ocupacionales a partir de la propia clasificación muy desagregada (en su caso, la CNO-68 a 3 dígitos). El procedimiento es algo penoso de elaborar y no permite la captura de un elemento importante de la relación entre los puestos de trabajo y las ocupaciones: no todos los que se dedican a una misma ocupación realizan el mismo tipo de tareas. Así, un trabajador cuya ocupación queda definida por un conjunto de tareas dado puede ocupar su tiempo entre diversos subconjuntos diferentes de éstas, o en distintos aspectos específicos del proceso de trabajo. En ocasiones ocurre que los empleos cambian sustancialmente de contenido debido a cambios en la tecnología aplicada en los procesos de trabajo, o en relación con la organización técnica o social de éstos.

En la literatura internacional, el desarrollo de tipo analítico más relevante tiene su origen en las clasificaciones utilizadas por el Departamento de Trabajo norteamericano. En este caso, el uso de la clasificación tipológica no es un sustituto de la clasificación de ocupaciones (una versión de la ISCO-88), sino un procedimiento para delimitar los tipos de tareas realizadas dentro de cada categoría ocupacional, que ocupa los dígitos cuatro a seis de la clasificación estándar (USDL, 1991). Tanto en esta versión más antigua como en las versiones españolas, el criterio básico de clasificación es la aclaración del objeto principal de las tareas propias de la ocupación, que se clasifica en tres tipos: operaciones sobre objetos, cosas o materiales; tratamiento de información y trato de personas.

Pero estos distintos aspectos de la ocupación se encuentran mezclados entre sí en la mayoría de las situaciones (Desrosieres y Thevenot, 1990). Varios ejemplos pueden ilustrar esto.

(a) Entre los profesionales superiores, se suele considerar como parte de la ocupación tanto el ejercicio de ésta como el conjunto de tareas de gestión ligadas a su control. De este modo, bajo el mismo epígrafe aparece tanto el médico especialista de un hospital, como el médico dedicado a la gestión hospitalaria (confundiendo así ocupación con oficio, o en este caso profesión), como el in-

\footnotetext{
${ }^{3}$ Como señala uno de los evaluadores de este artículo, es importante distinguir en este punto entre una clasificación propiamente dicha, que en principio utiliza un solo criterio para distinguir los particulares del fenómeno estudiado, de una tipología, que combina criterios diversos. Las «clasificaciones» de ocupaciones son en realidad tipologías impuras, en las cuales se aplican los criterios de clasificación de un modo no uniforme en las distintas partes de la taxonomía.
}

EMPIRIA. Revista de Metodología de Ciencias Sociales. N. ${ }^{\circ}$ 17, enero-junio, 2009, pp. 91-121. ISSN: 1139-5737 
vestigador, como el médico que ejerce su profesión como profesional liberal y dedica parte importante de su tiempo a la gestión de su consulta. En varios de estos casos, tenemos el problema añadido de que en el ejercicio de la profesión se realizan varias de estas tareas simultáneamente, y es complicado establecer un criterio para elegir una de ellas como tarea principal.

(b) Desde hace tiempo se sabe que las clasificaciones de ocupaciones no capturan de un modo adecuado la distinción entre los trabajadores y los supervisores (González, 1992a y 1992b), puesto que se clasifica a éstos últimos en la misma categoría que a los supervisados. Aquí el problema es grave, dado que en relación con determinadas características del empleo es necesario distinguir a los supervisores, y que esta distinción es muy relevante desde un punto de vista social. Técnicamente, es muy complicado establecer una frontera nítida entre las situaciones en las que el supervisor realiza las mismas tareas que los supervisados y las situaciones en las que no las realiza. Es más, en muchos puestos de supervisión la decisión sobre si el supervisor realiza el mismo trabajo que los supervisados es discrecional y exclusiva de éste.

(c) Una distinción que en su momento se consideró importante fue la que se establecía en el interior del trabajo administrativo entre los que realizan tareas exclusivamente administrativas y los que realizan tareas de atención al público. Por varias razones, el problema no es inocuo: el trabajo administrativo constituye la primera categoría en cuanto a número de efectivos prácticamente en cualquier clasificación de ocupaciones. Esto se debe tanto a su importancia numérica, como a la dificultad para establecer fronteras internas nítidas entre diversos aspectos de las tareas administrativas, como a la extensión y universalidad de la definición social de lo que es un «administrativo». Además, uno de los argumentos centrales sobre el cambio ocupacional fue en su momento la creciente importancia de las tareas de atención al público entre los administrativos (Garrido, 1991, pg. 30). La clasificación internacional de ocupaciones del año 1988 (ISCO-88) solucionó este problema distinguiendo a los administrativos en sentido estricto de los que realizan tareas de atención al público. Sin embargo, podemos pensar que hay otras distinciones relevantes: los que fundamentalmente asisten al trabajo de otros, los que tienen capacidad de decisión sobre el contenido de su trabajo y los que simplemente hacen funciones de trascripción y gatekeeping administrativo.

El uso de las clasificaciones estadigráficas habituales es suficiente para la mayoría de las tareas de investigación (O’Reilly y Rose, 1998; Thevenot, 1983; Collado y Martinez, 1995, pg. 338), especialmente en la medida en la que permiten su reagrupación en categorías con un contenido social comprensible. Sin embargo, en el análisis del cambio ocupacional y de las transformaciones en el trabajo necesitamos procedimientos más refinados, de tipo analítico, para examinar la operación interna de las ocupaciones. Hay dos buenas razones para analizar las tendencias en los contenidos reales de las ocupaciones. En primer lugar, porque estamos suponiendo que éstos tienen consecuencias sociales, pero no tenemos información que sirva para sustentar esta idea. El resultado es que gran

EMPIRIA. Revista de Metodología de Ciencias Sociales. N. ${ }^{\circ}$ 17, enero-junio, 2009, pp. 91-121. ISSN: 1139-5737 
parte del análisis ocupacional está basado en supuestos apriorísticos o en la experiencia directa, pero no en un análisis numérico formal de los contenidos de las ocupaciones. En segundo lugar, todo esto es importante en relación con los propios esfuerzos de clasificación ocupacional: en la medida en la que conozcamos en profundidad el funcionamiento de las ocupaciones y la existencia de inconsistencias internas en las categorías ocupacionales podremos mejorar el trabajo de tipologización que se encuentra en la base de gran parte del esfuerzo de investigación en ciencias sociales.

En este estudio se hace una aproximación empírica al problema. Es decir, en lugar de atribuir características a las ocupaciones en función de su definición nominal, utilizamos información diferente de la contenida en la propia clasificación para establecer las características de las ocupaciones, y comparamos los resultados con la clasificación propiamente dicha. Este ejercicio no está exento de dificultades, que se irán examinando a medida que se presenten. En la primera sección se explica el enfoque que adoptamos para analizar los contenidos de las ocupaciones. La propuesta consiste en centrar la atención en dos propiedades que distinguen unas tareas de otras dentro del paquete que conforma la ocupación: el objeto sobre el que se aplica, y la complejidad de las tareas que se realizan en el ejercicio de esa ocupación. Como expondremos, la propuesta que nos parece más convincente es el examen de la complejidad en función del objeto. En la segunda sección explicamos las características de nuestra fuente de datos, los problemas encontrados con las variables y la clasificación de ocupaciones utilizada. En la tercera examinamos la validez de nuestra estrategia de análisis de los objetos de las ocupaciones a partir de datos de encuesta. En la cuarta sección exponemos un intento de medir la complejidad de las ocupaciones según nuestros datos, para después ligar el examen de la complejidad con la cualificación requerida en el puesto y detenernos en algunos casos especialmente complicados. Por último, discutimos los resultados principales y proponemos líneas de trabajo futuras.

\section{OBJETO, CONTROL Y COMPLEJIDAD EN LAS OCUPACIONES}

En el análisis de los mercados de trabajo, y al margen de los distintos enfoques teóricos aplicados, se ha considerado la cualificación como el elemento explicativo central de las dinámicas laborales. Esto explica el que en las clasificaciones de ocupaciones domine la cualificación como elemento tipológico sobre cualquier otra consideración (Jones, 1985; González, 1992a; Collado y Martínez, 1995). En la ISCO-1968, por ejemplo, la cualificación dominaba la clasificación en grandes grupos ocupacionales, para después subdividir las categorías correspondientes según la profesión en los niveles más altos y según la rama de actividad en los más bajos. Un argumento fundamental para sostener una estrategia de este tipo es que la cualificación está ligada a la dificultad y a la especificidad de las tareas que se realizan. Estos aspectos de la ocupación, sin embargo, se hacen muy difíciles de medir. El resultado es que tenemos que trabajar con apro-

EMPIRIA. Revista de Metodología de Ciencias Sociales. N. ${ }^{\circ}$ 17, enero-junio, 2009, pp. 91-121. ISSN: 1139-5737 
ximaciones al nivel de cualificación de la fuerza de trabajo que no siempre son adecuadas a efectos de investigación (Green, 1999; Neilsen, 1986).

Pero las diferencias entre unas ocupaciones y otras no se refieren tan sólo al nivel de cualificación. Un examen detallado de los contenidos relevantes de las ocupaciones nos lleva a centrar la atención sobre otros aspectos de interés. En el marco de este análisis vamos a fijarnos en dos de ellos: el objeto al que se aplica el proceso de trabajo y el grado de complejidad exigida por la realización de las tareas que contiene.

El objeto al que se aplica una tarea configura en gran medida la propia tarea. Delimitando las diferentes tareas hacia las que se orientan las ocupaciones tenemos la oportunidad de realizar una distinción fundamental entre unos tipos de ocupaciones y otros. Garrido (1991) propone distinguir entre las ocupaciones que trabajan con objetos, las que trabajan con información y las que trabajan con personas. En el contexto de la investigación original se aplicó esta distinción por imputación a las categorías ocupacionales distinguidas a tres dígitos. Este trabajo mostró en su momento la creciente importancia numérica de las ocupaciones dedicadas al trato de personas. El objeto determina en gran medida el carácter social de la ocupación: la distinción clásica entre ocupaciones manuales y no manuales está basada en una distinción de los contenidos ocupacionales basada en el objeto.

Por su parte, la complejidad de tarea tiene varios aspectos, relacionados con la variedad de cosas que se deben hacer en un puesto de trabajo y con su dificultad intrínseca (sobre el concepto de complejidad ver Weaver, 1948). La complejidad atañe por lo tanto al tipo y cantidad de habilidades necesarias para desempeñar un trabajo. En realidad la cualificación requerida es un aspecto de la complejidad de la tarea. Por si misma, la complejidad de tarea es extremadamente difícil de medir: está ligada a la toma de decisiones en el desempeño de una tarea, a la cantidad, precisión y certidumbre necesarias para la toma de decisiones; a la propia complejidad cognitiva de la concepción de la tarea, al tiempo de entrenamiento necesario para desarrollar un desempeño adecuado y, en fin, al margen de acción en su realización.

Un problema fundamental en el análisis de la complejidad de las ocupaciones es el hecho de que los contenidos en tareas de las ocupaciones contienen tipos o modelos de complejidad muy diversos (Popping, 1995). Es habitual que en un puesto de trabajo concreto las tareas contengan una combinación heterogénea de habilidades y de acciones articuladas que responden a modelos aprendizaje, evaluación y toma de decisiones de distinto tipo. A modo de propuesta muy básica, el cuadro 1 presenta una reseña general de al menos siete tipos de complejidad que pueden estar presentes de un modo u otro en la realización de un conjunto de tareas dado. El eje sobre el que se estructura este análisis está referido al conjunto de tareas cognitivas -de aprendizaje, percepción, evaluación y acción- con las que se corresponden las habilidades. En la medida en la que cualquier tarea exige un mínimo de actividades cognitivas, está ligada a un modelo de aprendizaje o modo de adquisición de las habilidades correspondientes a esas tareas. La tercera columna representa ejemplos de esas habilidades.

EMPIRIA. Revista de Metodología de Ciencias Sociales. N. ${ }^{\circ}$ 17, enero-junio, 2009, pp. 91-121. ISSN: 1139-5737 
La adquisición de habilidades mecánicas y su aplicación es un buen primer ejemplo del tipo de dificultades que nos encontramos a la hora de establecer un patrón de análisis de los contenidos ocupacionales. Por una parte, el aprendizaje del uso de herramientas está ligado a la práctica con ellas. La comprensión de cómo se utiliza una herramienta manual rara vez lleva más de cinco minutos, pero el uso adecuado de las herramientas - de una llave inglesa, pongamos por caso- y la elección de la herramienta adecuada para cada tarea exige un proceso de aprendizaje largo y, en general, ligado a la experiencia en su uso. La habilidad para utilizarlas, además, está asociada a capacidades naturales que pueden facilitar su aprendizaje y uso, o por el contrario dificultarlos. Existen, en realidad, al menos dos tareas diferentes implicadas en el uso de herramientas mecánicas: la propia capacidad de uso de la herramienta y la capacidad de decisión de qué herramienta usar en una situación determinada. En un nivel posterior, se genera la necesidad de adaptar el uso de una herramienta dada para una tarea para la cual no ha sido diseñada. Todo este proceso cognitivo y mecánico implica una comprensión intuitiva de la densidad de la herramienta y sus capacidades, de los efectos causales de su uso y de las consecuencias de su forma.

Cuadro 1. Modelos de complejidad de tarea

\begin{tabular}{|c|c|c|}
\hline Tipo de complejidad & Modo de adquisición & Ejemplos \\
\hline & Primer nivel & \\
\hline $\begin{array}{l}\text { Desarrollo de habilidades } \\
\text { físicas y/o implícitas }\end{array}$ & Natural, entrenable & $\begin{array}{l}\text { El oído musical, la } \\
\text { atención, capacidades } \\
\text { cognitivas }\end{array}$ \\
\hline \multirow[t]{2}{*}{ Habilidades sociales } & Natural, entrenable & Vender \\
\hline & Segundo nivel & \\
\hline $\begin{array}{l}\text { Adquisición de habilidades } \\
\text { mecánicas }\end{array}$ & $\begin{array}{l}\text { Por tiempo y curva de } \\
\text { aprendizaje }\end{array}$ & Tocar un instrumento \\
\hline \multirow[t]{2}{*}{ Manipulación simbólica } & $\begin{array}{l}\text { Por tiempo y curva de } \\
\text { aprendizaje }\end{array}$ & Lectoescritura \\
\hline & Tercer nivel & \\
\hline $\begin{array}{l}\text { Capacidad para evaluar una } \\
\text { situación con información } \\
\text { escasa }\end{array}$ & Por experiencia & Encontrar una avería \\
\hline \multirow[t]{2}{*}{ Reconocimiento de patrones } & $\begin{array}{l}\text { Por experiencia } \\
\text { (formación experta) }\end{array}$ & Leer el tiempo \\
\hline & Cuarto nivel & \\
\hline $\begin{array}{l}\text { Capacidad para generar } \\
\text { una estructura o una forma } \\
\text { a partir de elementos } \\
\text { indiferenciados }\end{array}$ & Por resultados y contraste & Diseñar una pieza \\
\hline
\end{tabular}

FUENTE: Elaboración propia

EMPIRIA. Revista de Metodología de Ciencias Sociales. N. ${ }^{\circ}$ 17, enero-junio, 2009, pp. 91-121. ISSN: $1139-5737$ 
Otro ejemplo de un orden diferente nos lo presta el aprendizaje de un instrumento musical. En primer lugar, en este caso la operación física sobre la herramienta es sólo una parte, aunque importante, del conjunto de la tarea. Según el tipo de instrumento la adquisición de habilidades manuales suficientes para el manejo de la herramienta -el instrumento- puede variar mucho con el tiempo. Pero el uso de la herramienta implica además el aprendizaje de un sistema de relaciones abstractas entre sonidos que puede no resultar intuitivo sin un entrenamiento cognitivo previo -la adquisición del oído musical-, que según todos los indicios ocurre durante la infancia. Además, como cualquiera que toque un instrumento puede corroborar, el aprendizaje mecánico del instrumento no es suficiente para realizar la tarea adecuadamente: hace falta en este caso (1) el desarrollo de habilidades más o menos sublimes e inasibles para realizar la tarea del modo adecuado, habilidades que están ligadas a un proceso de aprendizaje largo y penoso; y (2) un desarrollo de habilidades mecánicas mucho más complejas relacionadas con la coordinación de mano, respiración, oído y comprensión de la respuesta del instrumento a la acción humana.

La propuesta presentada en el cuadro 1 intenta capturar estas dificultades de análisis. Un elemento esencial en el desarrollo de cualquier tipo de habilidad es la manera en la que se adquiere. La forma más básica de aprendizaje es la que ocurre durante el desarrollo infantil: el desarrollo de capacidades innatas, en principio para el establecimiento de capacidades cognitivas y de comunicación, después para la adquisición de competencias culturales básicas. Estas habilidades son entrenables, y se utilizan de hecho en el contexto de la realización de las tareas propias de muchos empleos. En un segundo nivel se encuentran las habilidades que implican aprendizaje de ciertas habilidades compuestas a lo largo de un periodo de tiempo más o menos limitado: estos aprendizajes suponen (1) la activación de conjuntos de habilidades básicas y (2) el aprendizaje dirigido básicamente por repetición de patrones preestablecidos. En un tercer nivel se agrega la necesidad de aplicar conocimientos teóricos elaborados -o modelos del mundo elaborados- en el proceso de aprendizaje, ligado inevitablemente a la adquisición de experiencia sobre el proceso al que se aplican los conocimientos y destrezas adquiridos. Por último, en un cuarto nivel se genera la posibilidad de generar formas o estructuras nuevas a partir de elementos indiferenciados, lo cual supone la generación de conocimientos o estados del mundo novedosos a partir de información anterior escasa. El cuarto nivel no es exclusivo de los trabajos profesionales más complejos; puede, de hecho, encontrarse entre trabajos manuales de carácter artesanal o cualificados. Sin embargo, se contradice con el principio de rutina asignado en general a este tipo de ocupaciones.

Cada nivel suplementario implica un grado más elevado de racionalización del aprendizaje y un alejamiento de los procesos naturales de pensamiento y aprendizaje, y por lo tanto un coste de adquisición más elevado. En un puesto de trabajo concreto se realizan una serie de tareas mediante el uso de herramientas, cuyo aprendizaje puede ser más o menos complejo. Sin embargo, el uso de herramientas por sí mismo no agota en absoluto ni la complejidad del

EMPIRIA. Revista de Metodología de Ciencias Sociales. N. ${ }^{\circ}$ 17, enero-junio, 2009, pp. 91-121. ISSN: 1139-5737 
empleo - las herramientas complicadas pueden ser usadas de un modo rutinarioni el desarrollo de las tareas propias del puesto de trabajo. De este modo, podríamos aplicar una clasificación en términos de la complejidad de las tareas -ligada, por supuesto, a la complejidad y modo de los aprendizajes- para el conjunto de la estructura ocupacional. Esta clasificación debería superponerse sólo en parte con los niveles de estudios, que están sometidos a una fuerte regulación institucional.

Si analizamos ejemplos concretos de ocupaciones, estos diferentes niveles de complejidad pueden interpretarse en torno a tres aspectos distintos de las tareas que se realizan en el desempeño de un trabajo. (1) Por una parte, se realiza un uso más o menos intenso de las habilidades naturales de las personas, como la fuerza, la capacidad espacial o la sociabilidad. En términos generales, se puede suponer que este uso está limitado a tareas de complejidad baja o media, y su papel en la configuración del sistema de cualificaciones se hace relevante en la medida en la que sirve de filtro de entrada a los puestos de trabajo. La capacidad de comunicación sirve, por ejemplo, en un amplio espectro de ocupaciones como elemento cualificatorio básico, desde maestros y administrativos a telefonistas, policías o camareros. Un aspecto importante de las habilidades naturales es el hecho de que están sujetas a un efecto de modelado cultural: la capacidad de desempeño en muchos de estos empleos está ligada a la adquisición de modelos de comportamiento y de relación que son específicos de contextos culturales concretos, y cuyos ejemplos se encuentran desde en la dureza de empleos antiguos, como la marinería o la minería, hasta en la de habilidades de comunicación interpersonal en nuestras sociedades.

(2) En segundo lugar, gran parte de la complejidad se refleja en el uso de herramientas y el desarrollo de procedimientos y protocolos de mayor o menor dificultad, siempre en el sobreentendido de que las herramientas implican tanto una complejidad interna -es decir, incluida en el propio diseño de los ordenadores- como una complejidad de uso. Determinadas herramientas son sencillas desde el punto de vista del diseño, pero complejas desde el punto de vista del manejo. Con otras ocurre lo contrario: una grúa o un teléfono presentan diseños complejos con un uso sencillo. En algunos casos, la complejidad del diseño incluye en sí misma la posibilidad tanto de un uso complejo como de uno sencillo, como es el caso de los ordenadores. Interpreto en este caso que los procedimientos organizativos son herramientas de uso colectivo (Scott, 1981/2003, pg. 33 y ss.).

(3) La tercera manifestación de la complejidad se da en los requisitos cognitivos de la realización de una tarea. Éstos son muy diversos y no incluyen únicamente la necesidad de acumular unos conocimientos dados para su realización, sino también la de procesar la información del entorno con procedimientos más o menos sofisticados y la de procesar el curso de la realización de la propia tarea. Esto puede implicar a su vez el desarrollo de heurísticas educables, bien por medio de la experiencia, bien por medio de un entrenamiento más o menos formal. No cabe duda de que tanto el uso de habilidades naturales como el de instru-

EMPIRIA. Revista de Metodología de Ciencias Sociales. N. ${ }^{\circ}$ 17, enero-junio, 2009, pp. 91-121. ISSN: 1139-5737 
mentos implica procesos cognitivos, pero en este caso estamos hablando de tareas cuya realización depende exclusivamente de una acción cognitiva.

Estos tres aspectos, -natural, procedimental y cognitivo- se manifiestan con distinto nivel de complejidad en cada una de las ocupaciones. Si hay una característica común en las transformaciones ocupacionales de las últimas décadas es el deslizamiento de la economía desde ocupaciones industriales hacia ocupaciones de servicios, tanto cualificadas como sin cualificar. Esto supone un paso desde ocupaciones con un componente procedimental fuerte hacia cualificaciones con un componente cognitivo y relacional (y por lo tanto natural) predominante. Camareros, dependientes y peluqueros necesitan de esas dotes naturales básicas para interactuar con los clientes. Por lo demás, los requisitos de tipo procedimental, relacionados con el manejo de herramentas y sistemas de trabajo son limitados. Estos dos aspectos del trabajo procedimental, por otra parte, muestran sus diferencias de un modo más marcado en el trabajo de los administrativos que implica un manejo sencillo de herramientas y un seguimiento complejo de procedimientos organizativos.

Para examinar estos aspectos de la estructura ocupacional, vamos a realizar un ejercicio analítico en dos pasos. En primer lugar, examinaremos la atribución que realizan los propios trabajadores de cual es el objeto principal de sus empleos. Compararemos a continuación esta medición con una distribución de ocupaciones convencional en el sentido de que está basada en el procedimiento clásico en tres pasos de (1) descripción del empleo por el encuestado del modo más concreto posible; (2) traducción al sistema de códigos por parte del equipo de campo; y (3) recodificación por parte del analista. Después pasaremos a comparar una medición de la complejidad, que se explica en la siguiente sección, con la misma clasificación de ocupaciones.

\section{DATOS}

En algunas fuentes de datos encontramos propuestas que puede aportar información de interés sobre los procedimientos de medición de la complejidad de tarea. El estudio 2634 del CIS, titulado «Clases sociales y estructura social», cuyo trabajo de campo fue realizado en el primer trimestre de 2006, nos brinda información abundante sobre el contenido en tareas de las ocupaciones, sobre las tareas de supervisión, sobre las características de las organizaciones en las que se desempeña y sobre la responsabilidad en el puesto de trabajo ${ }^{4}$. La encuesta

${ }^{4}$ El estudio CIS 2634 está basado en una muestra representativa de 8.265 personas, entrevistadas personalmente y seleccionadas con el procedimiento de muestreo polietápico habitual en los estudios del CIS: selección de las unidades primarias (secciones) por estratos de comunidad autónoma y tamaño del municipio, y de las secundarias (individuos) por aplicación de cuotas de sexo y edad calculadas en función de la tasa de ocupación y selección mediante rutas. Incluye una sobrerepresentación de la muestra para Andalucía, que ha sido ponderada en los análisis realizados en este estudio.

EMPIRIA. Revista de Metodología de Ciencias Sociales. N. ${ }^{\circ}$ 17, enero-junio, 2009, pp. 91-121. ISSN: 1139-5737 
contiene dos submuestras, una de ocupados y otra de no ocupados, cuya diferencia consiste en que los primeros responden a secciones del cuestionario a las que no deben responder los segundos. Nos importa en este caso que en el diseño muestral se procuró asegurar la representatividad estadística de los ocupados.

El cuestionario contiene específicamente cuatro preguntas que nos resultan de interés: en la primera se pregunta por el tipo de objeto al que se aplica el proceso de trabajo, con la siguiente formulación: «P2.16 ¿Qué tipo de actividades ocupan más tiempo en su trabajo: las dedicadas a tratar con objetos, con datos, o con personas? ¿Y en segundo lugar? ¿Y en tercer lugar?». Realizaremos en primer lugar un análisis de esta variable, como indicador del objeto principal al que se dirige la actividad realizada en la ocupación. El uso de la evaluación realizada por los propios entrevistados presenta algunas dificultades metodológicas. La principal es que estamos suponiendo que los encuestados pueden evaluar correctamente el objeto sobre el que se aplica su trabajo, lo cual es una tarea que supone en realidad una cierta dificultad. Baste por ahora con aceptar este procedimiento como alternativa a la atribución de objetos a las categorías ocupacionales previamente definidas, con la ventaja de que la información se refiere específicamente a cada individuo en su puesto de trabajo y no a categorías ocupacionales completas. Estamos aceptando un margen de error desconocido -el generado por el criterio de los propios encuestados- a cambio de otro -el generado por el investigador.

En la misma encuesta, un poco más adelante, se pregunta por el nivel de complejidad de las tareas realizadas en el puesto de trabajo, con la siguiente formulación: «Lea cuidadosamente esta tarjeta y dígame en cuál de los siguientes 11 niveles ubicaría las actividades manuales más complejas que realiza Vd. en su trabajo», sustituidas más tarde por actividades con datos y por actividades que realiza con otras personas. Como opciones de respuesta, se ofrecen las que se presentan en la columna derecha de los cuadros 2 a 4 .

Esta forma de medir la complejidad presenta algunos problemas de cierta seriedad. El principal es que la ordenación de los niveles de complejidad para un rango tan grande de niveles diferentes no es en general clara ni unívoca. El lector puede demostrar esto intentando organizar las categorías tal y como vienen dadas en el cuestionario, y observará dos cosas: la primera es que el número de categorías afecta al parecido entre la ordenación original en el cuestionario y la que genere. Es decir, cuantos más elementos posibles se puedan ordenar, mayor es la probabilidad de alcanzar un orden diferente del propuesto. El segundo problema es que la ordenación original presenta algunos criterios concretos que nos parecen dudosos. La instalación de maquinaria, por ejemplo, difícilmente va a resultar más compleja que la realización de trabajos de precisión. En este caso, tomar una decisión sobre el nivel de complejidad requeriría una medición directa que no estamos en disposición de hacer. La manipulación de procesadores de texto -es decir, las tareas manuales ligadas al manejo de las TIC- se puede considerar tanto una actividad compleja como simple, según se de más o menos peso a los aspectos más cognitivos de la tarea.

EMPIRIA. Revista de Metodología de Ciencias Sociales. N. ${ }^{\circ}$ 17, enero-junio, 2009, pp. 91-121. ISSN: $1139-5737$ 
Cuadro 2. Complejidad de las tareas manuales

\begin{tabular}{|c|c|c|}
\hline Respuestas & $\begin{array}{r}\% \text { de la } \\
\text { muestra }\end{array}$ & $\begin{array}{l}\text { Reordenación y reasignación de } \\
\text { grados de complejidad }\end{array}$ \\
\hline $\begin{array}{l}\text { Maneja unos pocos objetos, } \\
\text { con o sin ayuda de herramientas } \\
\text { sencillas }\end{array}$ & 27,4 & \multirow{2}{*}{$\begin{array}{l}1 \text { Desempeño de tareas manuales } \\
\text { básicas }\end{array}$} \\
\hline $\begin{array}{l}\text { Suministra, limpia o vacía } \\
\text { lugares o máquinas }\end{array}$ & 5,2 & \\
\hline $\begin{array}{l}\text { Vigila o controla maquinaria } \\
\text { fija y automatizada }\end{array}$ & 3,4 & \multirow[b]{2}{*}{$\begin{array}{l}2 \text { Manejo básico de máquinas con } \\
\text { interfaces dirigidos }\end{array}$} \\
\hline $\begin{array}{l}\text { Ejecuta tareas pre-programadas } \\
\text { con máquinas de oficina y caja, } \\
\text { como fotocopiadoras, lectores } \\
\text { ópticos, etc. }\end{array}$ & 4,1 & \\
\hline $\begin{array}{l}\text { Manipula objetos varios con } \\
\text { ayuda de máquinas-herramienta }\end{array}$ & 10,1 & $\begin{array}{l}3 \text { Manipulación de objetos y control } \\
\text { del comportamiento de herramientas } \\
\text { con interfaz abierto }\end{array}$ \\
\hline $\begin{array}{l}\text { Opera procesadores de texto } \\
\text { y otros programas de ordenador }\end{array}$ & 15,4 & 2 \\
\hline $\begin{array}{l}\text { Conduce y controla los mandos } \\
\text { de máquinas y de vehículos } \\
\text { móviles }\end{array}$ & 6,1 & $\begin{array}{l}3 \text { Manipulación de máquinas con } \\
\text { interfaz y uso abierto }\end{array}$ \\
\hline $\begin{array}{l}\text { Enciende, ajusta o pone a punto } \\
\text { maquinaria y equipamiento } \\
\text { móvil }\end{array}$ & 1,4 & $\begin{array}{l}4 \text { Evaluación y ajuste del } \\
\text { funcionamiento de máquinas. }\end{array}$ \\
\hline $\begin{array}{l}\text { Realiza trabajos de precisión } \\
\text { directamente o con máquinas- } \\
\text { herramientas }\end{array}$ & 4,2 & $\begin{array}{l}5 \text { Tareas de manipulación compleja, } \\
\text { que implica tiempos de aprendizaje } \\
\text { elevados, uso combinado de varias } \\
\text { herramientas o evaluación de los } \\
\text { movimientos realizados. }\end{array}$ \\
\hline $\begin{array}{l}\text { Instala o repara máquinas o } \\
\text { equipamientos }\end{array}$ & 2,1 & \multirow{2}{*}{4} \\
\hline $\begin{array}{l}\text { Maniobra maquinaria pesada y } \\
\text { equipamiento complejo }\end{array}$ & 0,9 & \\
\hline
\end{tabular}

FUENTE: CIS 2634 y elaboración propia

Estas dificultades implican la necesidad de replantear la clasificación de las tareas realizadas en el puesto en cuanto a los niveles de complejidad que conllevan. Se ha abordado este problema con una estrategia doble. Por una parte, se ha generado una reordenación de los niveles de complejidad planteados por los ítems originales según un criterio propio; por otro lado, se han reducido los ni- 
veles presentados por las tarjetas de encuesta originales a cinco posibilidades en cada uno de los tres ítems, tal y como viene reflejado en los cuadros 2 a 4 . La columna central de cada uno de los cuadros presenta la proporción de los que responden que se ubica en cada una de las categorías originales. Al pie del cuadro se encuentra la proporción total de la población ocupada que afirma realizar alguna de las actividades consignadas en la pregunta: un $80 \%$ realiza actividades manuales de algún tipo, un $56 \%$ trabaja en algún momento con información (en un sentido específico e inmediato), y un $90 \%$ considera que trabaja con personas de un modo u otro.

El procedimiento de imputación, por lo tanto, consiste en reordenar las categorías de un modo que contenga la menor cantidad posible de sospechas con respecto a su ordenabilidad, para después asignar un valor de 1 a 5 a cada uno de los niveles generados de este modo. Esta cifra es convencional y no representa distancias reales entre distintos niveles de complejidad de las tareas, pero nos

Cuadro 3. Complejidad de las tareas informacionales

\begin{tabular}{|c|c|c|}
\hline Respuestas & $\begin{array}{c}\text { \% de la } \\
\text { muestra }\end{array}$ & $\begin{array}{l}\text { Reordenación y reasignación de } \\
\text { grados de complejidad }\end{array}$ \\
\hline $\begin{array}{l}\text { Ordena o clasifica cosas o } \\
\text { datos siguiendo instrucciones } \\
\text { precisas }\end{array}$ & 11,9 & \multirow{2}{*}{$\begin{array}{l}1 \text { Operaciones que exigen comprensión } \\
\text { y manipulación simbólicas básicas }\end{array}$} \\
\hline $\begin{array}{l}\text { Copia, transcribe, o lee datos } \\
\text { y símbolos }\end{array}$ & 3,8 & \\
\hline $\begin{array}{l}\text { Cuenta o interpreta resultados } \\
\text { numéricos, esquemas o } \\
\text { diagramas }\end{array}$ & 4,6 & \multirow{2}{*}{$\begin{array}{l}2 \text { Operaciones que implican la } \\
\text { aplicación de un conocimiento previo } \\
\text { a información dada }\end{array}$} \\
\hline $\begin{array}{l}\text { Recopila y clasifica cosas o } \\
\text { datos siguiendo, en parte, su } \\
\text { propio criterio }\end{array}$ & 6,1 & \\
\hline $\begin{array}{l}\text { Analiza y evalúa datos, según } \\
\text { criterios o normas conocidas }\end{array}$ & 10,6 & \multirow[b]{2}{*}{$\begin{array}{l}3 \text { Generación de estados } \\
\text { informacionales nuevos a partir de } \\
\text { conocimiento previo }\end{array}$} \\
\hline $\begin{array}{l}\text { Crea o adapta diseños, métodos } \\
\text { y procesos a necesidades } \\
\text { concretas, aplicando recetas o } \\
\text { principios conocidos }\end{array}$ & 6,4 & \\
\hline $\begin{array}{l}\text { Coordina actividades o } \\
\text { planifica sus objetivos y } \\
\text { establece métodos para } \\
\text { evaluarlas y revisarlas }\end{array}$ & 10 & $\begin{array}{l}4 \text { Generación de estados nuevos en } \\
\text { condiciones de incertidumbre }\end{array}$ \\
\hline $\begin{array}{l}\text { Sintetiza información compleja } \\
\text { proponiendo nuevos enfoques }\end{array}$ & 2,7 & 5 Generación de conocimiento \\
\hline
\end{tabular}

FUENTE: CIS 2634 y elaboración propia

EMPIRIA. Revista de Metodología de Ciencias Sociales. N. ${ }^{\circ}$ 17, enero-junio, 2009, pp. 91-121. ISSN: 1139-5737 
permite realizar operaciones de cuantificación sobre la información y, sobre todo, nos permite comparar niveles de complejidad en distintos aspectos de cada ocupación y entre ocupaciones. Téngase en cuenta que vamos a realizar un uso forzado del indicador, puesto que se trata de una medida ordinal de la complejidad de tarea y no de una medición cuantitativa de ésta en términos de grados de libertad, requisitos de cómputo u otras posibilidades, que no pueden pasar por ahora de un estado teórico.

La función principal de las variables resultantes es servir como indicadores numéricos de los niveles de complejidad de las tareas realizadas en el puesto de trabajo. Para medir la complejidad total, se presentan dos propuestas alternativas. La primera consiste en sumar las puntuaciones de cada una de las tres dimensiones del objeto de trabajo. De este modo obtenemos un indicador único de la complejidad de tarea de la ocupación de cada individuo, pero ocultamos en gran medida el hecho de que las diferentes dimensiones de ésta son disimilares entre sí. La segunda posibilidad es ponderar esa suma de modo que otorguemos a cada uno de los tres componentes un peso específico en función de un criterio previo, arbitrario en el sentido de que el factor de ponderación sólo puede ser un reflejo de la importancia relativa dada a cada una de las tres dimensiones. En esta investigación se ha decidido asignar dos puntos a la complejidad de las tareas informacionales y tres puntos a la complejidad de las tareas personales. La razón principal es que consideramos que la complejidad cognitiva de las relaciones de interacción es mayor a la de la resolución de problemas aislados, que a su vez es mayor que la requerida para desarrollar habilidades manuales. Una versión menos arriesgada pero basada en la misma idea (la de que la complejidad cognitiva de las tareas de manejo de información y de trato con personas es superior a la del trabajo manual) implicaría asignar un punto de ponderación a la puntuación de complejidad en las tareas manuales, dos puntos al trato de personas y otros dos puntos al trato con información. Los resultados son muy similares a los que tenemos en este caso y, para trabajar con los datos agregados, son relativamente intercambiables. En cualquier caso, la asociación entre las puntuaciones de cada una de las tres dimensiones indica que mientras la complejidad en el trabajo manual es por completo independiente de las otras dos dimensiones (con correlaciones de 0,054 y -0,052), la asociación entre trabajo informacional y de trato con personas es relativamente elevada $(0,48)$, siempre teniendo en cuenta que la mayor parte de las ocupaciones permanecen en el primer nivel de complejidad en el trabajo con información.

Con el objetivo de analizar los resultados de esta medición se utiliza una clasificación de ocupaciones compactada en 21 categorías que ya se ha presentado en otras ocasiones (Rivière, 2004) y que llamaremos Clasificación Polivalente de Ocupaciones (CPO). Sus principales características son (1) la distinción de las ocupaciones profesionales en términos de su ámbito de actuación, entre profesiones de los servicios (básicamente sanidad y educación), profesiones aplicadas (orientadas a la creación de cosas: ingeniería y arquitectura, por ejemplo) y las profesiones de regulación institucional (orientadas a la gestión de procesos de

EMPIRIA. Revista de Metodología de Ciencias Sociales. N. ${ }^{\circ}$ 17, enero-junio, 2009, pp. 91-121. ISSN: 1139-5737 
tipo diverso en las organizaciones; por ejemplo: abogados). (2) La especificación de un grupo de semiprofesiones que incluye a todas aquellas categorías ocupacionales en las cuales, pese a no ser imprescindible la adquisición de una cualificación profesional específica o tener ésta un efecto limitado en el mercado, implica el uso de nuevas tecnologías o de aparatos técnicos complejos (informáticos de nivel bajo, fotógrafos, cameramen), junto a profesiones semi-

Cuadro 4. Complejidad de las tareas de trato de personas

\begin{tabular}{|c|c|c|}
\hline Respuestas & \begin{tabular}{|c|}
$\%$ de la \\
muestra
\end{tabular} & $\begin{array}{l}\text { Reordenación y reasignación de } \\
\text { grados de complejidad }\end{array}$ \\
\hline $\begin{array}{l}\text { Recibe instrucciones y } \\
\text { explicaciones sencillas }\end{array}$ & 21 & \multirow{3}{*}{1 Interacción básica } \\
\hline $\begin{array}{l}\text { Sirve a clientes, atendiendo sus } \\
\text { necesidades }\end{array}$ & $\mathbf{3 1 , 3}$ & \\
\hline $\begin{array}{l}\text { Intercambia información } \\
\text { corriente en el curso de trabajos } \\
\text { sencillos }\end{array}$ & 4 & \\
\hline $\begin{array}{l}\text { Ofrece información al público } \\
\text { u otros compañeros }\end{array}$ & 5 & \multirow{2}{*}{$\begin{array}{l}2 \text { Comunicación de estados y } \\
\text { situaciones }\end{array}$} \\
\hline $\begin{array}{l}\text { Vende un producto o servicio, } \\
\text { o defiende un punto de vista }\end{array}$ & 6,6 & \\
\hline $\begin{array}{l}\text { Entrena a individuos a actuar } \\
\text { de maneras concretas }\end{array}$ & 1,5 & \multirow{2}{*}{3 Comunicación empática } \\
\hline $\begin{array}{l}\text { Entretiene o divierte a personas } \\
\text { o audiencias }\end{array}$ & 1,4 & \\
\hline $\begin{array}{l}\text { Asesora, ofreciendo } \\
\text { conocimiento técnicos }\end{array}$ & 4,7 & \multirow[b]{2}{*}{$\begin{array}{l}4 \text { Comunicación orientada al control, } \\
\text { del comportamiento de otros }\end{array}$} \\
\hline $\begin{array}{l}\text { Instruye y enseña a otros a } \\
\text { alcanzar capacitaciones } \\
\text { profesionales }\end{array}$ & 4,6 & \\
\hline $\begin{array}{l}\text { Trata, diagnostica y cura a } \\
\text { personas o animales }\end{array}$ & 2,1 & 5 \\
\hline Supervisa a otros trabajadores & 3,8 & 4 \\
\hline Negocia acuerdos contractuales & 1,4 & \multirow{3}{*}{$\begin{array}{l}5 \text { Comportamiento racional complejo } \\
\text { orientado a la manipulación de } \\
\text { comportamientos, dependiendo de } \\
\text { teorías sobre estados sistémicos }\end{array}$} \\
\hline $\begin{array}{l}\text { Orienta a individuos con } \\
\text { problemas hacia nuevas metas. }\end{array}$ & 2 & \\
\hline $\begin{array}{l}\text { Lidera a otros, imponiendo su } \\
\text { visión sobre el objetivo a } \\
\text { cumplir y la estrategia para } \\
\text { alcanzarlo }\end{array}$ & $\mathbf{1 , 6}$ & \\
\hline
\end{tabular}

FUENTE: CIS 2634 y elaboración propia

EMPIRIA. Revista de Metodología de Ciencias Sociales. N. ${ }^{\circ}$ 17, enero-junio, 2009, pp. 91-121. ISSN: $1139-5737$ 
técnicas de los servicios (distintas de las descritas por Etzioni, 1969). (3) La distinción entre trabajos administrativos orientados al público y trabajos orientados al procesamiento de expedientes. (4) La especificación detallada de cinco tipos diferentes de ocupaciones de servicios de cualificación media o baja. Y (5) la distinción de cinco categorías diferentes en el ámbito del trabajo manual (Ver anexo).

\section{LOS COMPONENTES DE LA OCUPACIÓN}

El cuadro 5 nuestra la proporción de ocupados de cada una de las categorías ocupacionales que afirma que en su empleo realizan como tarea más importante el trato a personas, el de cosas o el de información. La tercera fila de cada categoría muestra los resultados en términos de residuos tipificados ajustados ${ }^{5}$. Los resultados son en general coherentes con los valores esperados. En general, los grupos de profesionales están divididos en dos grupos: los que trabajan básicamente con información y los que trabajan con personas. Los primeros están acumulados en las ocupaciones de profesionales aplicados, mientras los segundos lo están en los profesionales de los servicios sociales. Los profesionales medios, que son mayoritariamente enfermeras y maestros, están claramente decantados hacia el trabajo de trato con personas. Los trabajos de gerencia, dirección y supervisión suponen sobre todo trato de personas, y así aparece en nuestros datos: el 75,6\% de los directivos y gerentes, el 67,6\% de los supervisores administrativos y el $45,7 \%$ de los supervisores manuales considera que trabaja con personas como tarea principal.

La clasificación sólo consigue captar en parte la distinción entre los trabajadores administrativos que sólo procesan expedientes y los que atienden a las personas a las que afectan esos expedientes. Esto se debe a que el trabajo administrativo es especialmente indiferenciado, de tal modo que (1) las tareas varían de acuerdo con las necesidades concretas de gestión en cada momento, y (2) no hay una ruptura entre un tipo de funciones y otras. Podemos considerar que la clasificación de ocupaciones, de cualquier modo, subestima la cantidad de trabajadores administrativos que atienden al público. Por otra parte, es fácil para un trabajador administrativo que atiende al público proporcionando información entienda que la tarea que realiza es distribuir información, y no tratar con las personas de un modo prioritario.

En las ocupaciones de nivel intermedio (ventas y representación, seguridad, y revisión), así como en los servicios personales, es más elevada la pro-

\footnotetext{
${ }^{5}$ Es decir, de la razón entre el residuo y su error típico. Esta cifra se puede leer en unidades $\mathrm{Z}$ de desviación típica, de tal modo que para cada casilla sabemos si la diferencia entre el valor esperado y el observado es significativa en el caso de que el valor de la diferencia entre los residuos ajustados sea superior a un valor de referencia que varía en función del nivel de confianza: supongamos que 1,96 para un nivel de confianza del $95 \%$.
}

EMPIRIA. Revista de Metodología de Ciencias Sociales. N. ${ }^{\circ}$ 17, enero-junio, 2009, pp. 91-121. ISSN: 1139-5737 
porción de trabajadores que consideran que su tarea principal consiste en tratar con personas, incluso aunque estas tareas no contengan labores especialmente complejas. Son, en su conjunto, ocupaciones que tienen un fuerte componente de seguimiento y control de los comportamientos de otras personas, y que incorporan por lo tanto aspectos comunicativos como un componente esencial de éstas. En los servicios personales, de hecho, la definición de la categoría ocupacional incorpora el trabajo con personas como elemento diferenciador de otros grupos ocupacionales. En todas ellas, entre el 70 y el $80 \%$ de los encuestados afirma que su ocupación está ligada básicamente al trato a personas. Los servicios sin cualificación, por otra parte, presentan un elevado nivel de heterogeneidad interna -aunque también la mitad de los encuestados afirma trabajar básicamente con personas-. En este caso, podemos suponer que a causa de la inclusión de grupos ocupacionales muy diferentes entre sí, unidos tan sólo por no requerir cualificaciones generales ni específicas elevadas.

Cuadro 5. Tipo de tarea más importante según la ocupación

\begin{tabular}{|c|c|c|c|c|}
\hline Categoría ocupacional & Objetos & Datos & Personas & Casos totales \\
\hline \multirow{3}{*}{ DirGerent } & 25 & 7 & 99 & \multirow{3}{*}{131} \\
\hline & $19.1 \%$ & $5.3 \%$ & $75.6 \%$ & \\
\hline & -5.4 & -2.7 & 7.3 & \\
\hline \multirow[t]{3}{*}{ Emplea+10 } & 7 & 15 & 40 & \multirow[t]{3}{*}{62} \\
\hline & $11.3 \%$ & $24.2 \%$ & $64.5 \%$ & \\
\hline & -5.0 & 2.6 & 3.2 & \\
\hline \multirow{3}{*}{ ProfAplic } & 3 & 45 & 15 & \multirow[t]{3}{*}{63} \\
\hline & $4.8 \%$ & $71.4 \%$ & $23.8 \%$ & \\
\hline & -6.1 & 13.7 & -3.3 & \\
\hline \multirow[t]{3}{*}{ ProfRInst } & 4 & 27 & 55 & \multirow[t]{3}{*}{86} \\
\hline & $4.7 \%$ & $31.4 \%$ & $64.0 \%$ & \\
\hline & -7.1 & 5.0 & 3.7 & \\
\hline \multirow[t]{3}{*}{ ProfServs } & 17 & 30 & 213 & \multirow[t]{3}{*}{260} \\
\hline & $6.5 \%$ & $11.5 \%$ & $81.9 \%$ & \\
\hline & -12.0 & -.8 & 12.5 & \\
\hline \multirow[t]{3}{*}{ Prof Medio } & 10 & 52 & 181 & \multirow[t]{3}{*}{243} \\
\hline & $4.1 \%$ & $21.4 \%$ & $74.5 \%$ & \\
\hline & -12.4 & 3.9 & 9.7 & \\
\hline \multirow[t]{3}{*}{ Semiprofe } & 60 & 43 & 77 & \multirow[t]{3}{*}{180} \\
\hline & $33.3 \%$ & $23.9 \%$ & $42.8 \%$ & \\
\hline & -2.5 & 4.3 & -.5 & \\
\hline \multirow[t]{3}{*}{ PeqProCom } & 62 & 9 & 61 & \multirow[t]{3}{*}{132} \\
\hline & $47.0 \%$ & $6.8 \%$ & $46.2 \%$ & \\
\hline & 1.1 & -2.2 & .4 & \\
\hline \multirow[t]{3}{*}{ SupAdmCom } & 20 & 24 & 92 & \multirow[t]{3}{*}{136} \\
\hline & $14.7 \%$ & $17.6 \%$ & $67.6 \%$ & \\
\hline & -6.6 & 1.5 & 5.5 & \\
\hline
\end{tabular}

EMPIRIA. Revista de Metodología de Ciencias Sociales. N. ${ }^{\circ}$ 17, enero-junio, 2009, pp. 91-121. ISSN: $1139-5737$ 


\begin{tabular}{|c|c|c|c|c|}
\hline Categoría ocupacional & Objetos & Datos & Personas & Casos totales \\
\hline \multirow{3}{*}{ Administr } & 44 & 185 & 135 & 364 \\
\hline & $12.1 \%$ & $50.8 \%$ & $37.1 \%$ & \\
\hline & -12.2 & 22.2 & -3.03 & \\
\hline \multirow[t]{3}{*}{ Mostrador } & 16 & 24 & 60 & 100 \\
\hline & $16.0 \%$ & $24.0 \%$ & $60.0 \%$ & \\
\hline & -5.4 & 3.2 & 3.1 & \\
\hline \multirow{3}{*}{ VentasRep } & 65 & 29 & 238 & 332 \\
\hline & $19.6 \%$ & $8.7 \%$ & $71.7 \%$ & \\
\hline & -8.7 & -2.5 & 10.4 & \\
\hline \multirow[t]{3}{*}{ SegurRevi } & 17 & 10 & 70 & 97 \\
\hline & $17.5 \%$ & $10.3 \%$ & $72.2 \%$ & \\
\hline & -5.0 & -.9 & 5.5 & \\
\hline \multirow[t]{3}{*}{ ServPersn } & 66 & 3 & 264 & 333 \\
\hline & $19.8 \%$ & $.9 \%$ & $79.3 \%$ & \\
\hline & -8.6 & -6.9 & 13.3 & \\
\hline \multirow[t]{3}{*}{ ServNCual } & 24 & 5 & 34 & 63 \\
\hline & $38.1 \%$ & $7.9 \%$ & $54 \%$ & \\
\hline & -.7 & -1.2 & 1.5 & \\
\hline \multirow{3}{*}{ Limpieza } & 208 & 1 & 67 & 276 \\
\hline & $75.4 \%$ & $.4 \%$ & $24.3 \%$ & \\
\hline & 11,5 & $-6,5$ & -7.0 & \\
\hline \multirow{3}{*}{ SupervMan } & 19 & 6 & 21 & 46 \\
\hline & $41.3 \%$ & $13.0 \%$ & $45.7 \%$ & \\
\hline & -.1 & .0 & .2 & \\
\hline \multirow[t]{3}{*}{ Artesanos } & 78 & 3 & 20 & 101 \\
\hline & $77.2 \%$ & $3.0 \%$ & $19.8 \%$ & \\
\hline & 7.2 & -3.1 & -5.1 & \\
\hline \multirow{3}{*}{ ManCualif } & 433 & 18 & 43 & 494 \\
\hline & $87.7 \%$ & $3.6 \%$ & $8.7 \%$ & \\
\hline & 21.8 & -6.7 & -17.1 & \\
\hline \multirow[t]{3}{*}{ OperMaqCa } & 251 & 12 & 52 & 315 \\
\hline & $79.7 \%$ & $3.8 \%$ & $16.5 \%$ & \\
\hline & 14.0 & -5.1 & -10.4 & \\
\hline \multirow[t]{3}{*}{ ManNoCual } & 170 & 5 & 19 & 194 \\
\hline & $87.6 \%$ & $2.6 \%$ & $9.8 \%$ & \\
\hline & 13.1 & -4.5 & -10.0 & \\
\hline \multirow[t]{5}{*}{ Agricolas } & 173 & 2 & 13 & 188 \\
\hline & $92.0 \%$ & $1.1 \%$ & $6.9 \%$ & \\
\hline & 14.1 & -5.0 & -10.6 & \\
\hline & 1772 & 555 & 1869 & 4196 \\
\hline & $42.2 \%$ & $13.2 \%$ & $44.5 \%$ & \\
\hline
\end{tabular}

FUENTE: CIS 2634y elaboración propia.Ocupados. 
Entre los trabajadores manuales de distinto tipo los resultados son completamente consecuentes con la definición de las categorías ocupacionales. Aquellos que según la clasificación de ocupaciones se identifican como supervisores manuales se reparten a partes iguales entre los que consideran que trabajan con objetos y los que consideran que trabajan con personas; sin embargo, estos resultados son difícilmente significativos. Recordemos que las clasificaciones de ocupaciones subestiman de un modo importante la proporción de trabajadores dedicados a supervisión, y que tan sólo aparecen como un 1\% del conjunto de la población ocupada. Los resultados para el resto de los trabajadores manuales sí que son significativos, incluso con niveles de confianza del 99\%. Entre manuales cualificados y no cualificados y operarios de maquinaria encontramos tasas de respuesta afirmativa al trabajo con objetos de entre el 80 y el $90 \%$.

$\mathrm{El}$ acierto de este procedimiento de análisis se puede comprobar examinando las ocupaciones más numerosas. El cuadro 6 muestra las 23 categorías ocupacionales de la clasificación inicial (la CNO) desagregada a tres dígitos con mayor número de casos en nuestra fuente, ordenadas según el tamaño. Se han elegido todas aquellas que tuvieran más de 50 casos, y en conjunto representan un $51,7 \%$ de la fuerza de trabajo (lo cual quiere decir que la mitad de la fuerza de trabajo está concentrada en el 13,67\% de las categorías en las que hay algún caso en nuestros datos). A causa del nivel de desagregación, esta tabla nos indica al menos tres cosas diferentes: (a) el nivel de consenso dentro de la ocupación sobre el objeto de su proceso de trabajo tal y como se define habitualmente, (b) la diversidad interna de la categoría ocupacional en términos de las diferencias en lo referido a la realización de tareas, y (c) la claridad de la cuestión presentada en el cuestionario para los encuestados. Contando con el riesgo que supone trabajar con categorías ocupacionales que tienen tan pocos casos, se ha incluido una columna que informa sobre el error muestral para una muestra del tamaño de la categoría, con un nivel de confianza del 95\%, para el caso en el que la dispersión alcanzara su máximo (en el que el 50\% de los casos estuviera en una de las opciones). Este error es algo superior al $6 \%$ por encima o por debajo del dato en el caso de las categorías más numerosas y de un $\pm 13,86 \%$ para las categorías más pequeñas: debemos utilizarlo para hacernos una idea del margen de error conjunto en la categoría. El lector podrá calcular por sí mismo el error de cada una de las mediciones.

Casi todas las categorías mostradas en la tabla pueden interpretarse claramente según una de las lecturas ofrecidas más arriba. Varios grupos muestran niveles de acuerdo muy elevados por parte de los encuestados en lo relacionado con el objeto principal al que se aplica su proceso de trabajo. Es el caso, para el trato de personas, de docentes de los diferentes niveles [222 y 281], enfermeros [272], de representantes de comercio [332] y de camareros, bármanes y similares [502]. En todas estas categorías el acuerdo en cuanto al objeto principal de las tareas es completo, y superior al $80 \%$, y en los docentes y enfermeros el acuerdo es prácticamente total. También son varias las categorías con niveles de acuerdo superiores al $80 \%$ entre los que afirman que el objeto básico de sus tareas son objetos o materiales, y especialmente elevado entre peones de la cons-

EMPIRIA. Revista de Metodología de Ciencias Sociales. N. ${ }^{\circ}$ 17, enero-junio, 2009, pp. 91-121. ISSN: 1139-5737 
trucción [960], agricultores [601 y 602] y peones de industrias manufactureras. En todas estas categorías podemos considerar las respuestas a las otras dos opciones como errores de medición, aunque en algunos casos la presencia del trato a personas como tarea principal puede deberse a la presencia de supervisores ocultos, que reparten sus esfuerzos entre la realización de la tarea propiamente dicha y la supervisión del equipo de trabajo.

Cuadro 6. Ocupaciones más numerosas y tipo de tarea

\begin{tabular}{|c|c|c|c|c|c|}
\hline Ocupación & Objetos & Datos & Personas & Casos & Error \\
\hline 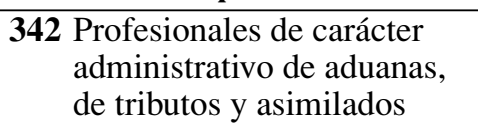 & 8.5 & 57.7 & 33.9 & 248 & \pm 6.24 \\
\hline $\begin{array}{l}533 \text { Dependientes y exhibidores } \\
\text { en tiendas, almacenes, } \\
\text { quioscos y mercadillos }\end{array}$ & 24.0 & 3.6 & 72.4 & 225 & \pm 6.55 \\
\hline 711 Albañiles y mamposteros & 87.4 & 0.6 & 12.0 & 167 & \pm 7.61 \\
\hline $\begin{array}{l}912 \text { Personal de limpieza de } \\
\text { oficinas, hoteles y otros } \\
\text { trabajadores asimilados }\end{array}$ & 90.3 & 0.7 & 9.0 & 144 & \pm 8.20 \\
\hline $\begin{array}{l}502 \text { Camareros, bármanes y } \\
\text { asimilados }\end{array}$ & 13.7 & 0.0 & 86.3 & 139 & \pm 8.34 \\
\hline 911 Empleados del hogar & 58.6 & 0.0 & 41.4 & 133 & \pm 8.53 \\
\hline $\begin{array}{l}222 \text { Profesores de enseñanza } \\
\text { secundaria }\end{array}$ & 1.8 & 0.9 & 97.2 & 109 & \pm 9.43 \\
\hline $\begin{array}{l}281 \text { Profesores de enseñanza } \\
\text { primaria e infantil }\end{array}$ & 1.1 & 0.0 & 98.9 & 89 & \pm 10.45 \\
\hline $\begin{array}{l}761 \text { Mecánicos y ajustadores } \\
\text { de maquinaria }\end{array}$ & 87.1 & 4.7 & 8.2 & 85 & \pm 10.69 \\
\hline $\begin{array}{l}602 \text { Trabajadores cualificados } \\
\text { por cuenta ajena en } \\
\text { explotaciones agrícolas }\end{array}$ & 92.7 & 0.0 & 7.3 & 82 & \pm 10.89 \\
\hline $\begin{array}{l}152 \text { Gerencia de empresas de } \\
\text { comercio al por menor sin } \\
\text { asalariados }\end{array}$ & 20.0 & 1.3 & 78.8 & 80 & \pm 11.03 \\
\hline $\begin{array}{l}970 \text { Peones de industrias } \\
\text { manufactureras }\end{array}$ & 91.1 & 2.5 & 6.3 & 79 & \pm 11.10 \\
\hline $\begin{array}{l}\text { 601 Trabajadores cualificados } \\
\text { por cuenta propia en } \\
\text { explotaciones agrícolas }\end{array}$ & 94.5 & 1.4 & 4.1 & 73 & \pm 11.55 \\
\hline $\begin{array}{l}332 \text { Representantes de comercio } \\
\text { y técnicos de ventas }\end{array}$ & 10.4 & 6.0 & 83.6 & 67 & \pm 12.06 \\
\hline $\begin{array}{l}980 \text { Peones del transporte y } \\
\text { descargadores }\end{array}$ & 77.3 & 3.0 & 19.7 & 66 & \pm 12.16 \\
\hline
\end{tabular}

EMPIRIA. Revista de Metodología de Ciencias Sociales. N. ${ }^{\circ}$ 17, enero-junio, 2009, pp. 91-121. ISSN: $1139-5737$ 


\begin{tabular}{lccccc}
\hline \multicolumn{1}{c}{ Ocupación } & Objetos & Datos & Personas & Casos & Error \\
\hline $\mathbf{2 7 2}$ Enfermeros & 4.9 & 0.0 & 95.1 & 61 & \pm 12.65 \\
\hline $\begin{array}{l}\mathbf{1 1 3} \text { Dirección de áreas y } \\
\text { departamentos } \\
\text { especializados }\end{array}$ & 7.3 & 41.8 & 50.9 & 55 & \pm 13.34 \\
\hline $\mathbf{8 6 2}$ Conductores de autobuses & 81.8 & 0.0 & 18.2 & 55 & \pm 13.34 \\
\hline $\mathbf{5 0 1}$ Cocineros y otros \\
$\begin{array}{l}\text { preparadores de comidas } \\
\mathbf{7 2 3} \text { Electricista de construcción } \\
\text { y asimilados }\end{array}$ & 70.4 & 1.9 & 27.8 & 54 & \pm 13.46 \\
\hline $\begin{array}{l}\text { Trabajadores de la industria } \\
\text { de la alimentación, bebidas }\end{array}$ & 78.8 & 17.3 & 3.8 & 52 & \pm 13.72 \\
$\quad$ y tabaco & 55.8 & 1.9 & 42.3 & 52 & \pm 13.72 \\
\hline $\mathbf{9 6 0}$ Peones de la construcción & 96.2 & 1.9 & 1.9 & 52 & \pm 13.72 \\
\hline $\begin{array}{l}\mathbf{1 2 2} \text { Gerencia de empresas de } \\
\text { comercio al por menor con } \\
\text { menos de 10 asalariados }\end{array}$ & 13.7 & 7.8 & 78.4 & 51 & \pm 13.86 \\
\hline
\end{tabular}

FUENTE: CIS 2634 y elaboración propia. Ocupados.

Este es claramente el caso de algunas de las categorías en las que el nivel de acuerdo no es tan elevado: entre peones del transporte y descargadores [980] hay una proporción importante de trabajadores que afirman trabajar prioritariamente con personas, así como entre los cocineros [501], para los que casi el $28 \%$ de los encuestados afirma trabajar con personas. No obstante, en este último caso parece razonable mantener algunas dudas. Este resultado parece significativo, ya que implica que estos trabajadores han considerado que el objeto de su trabajo son sus clientes y comensales, o bien sus subordinados.

Algunas de las categorías merecen una atención especial. Los administrativos [342], en los que en esta encuesta se han incluido empleados de aduanas y de tributos, presentan cerca de un $34 \%$ de casos que afirma trabajar con personas. Esta diversidad interna no puede atribuirse a la presencia de supervisores ocultos, sino al hecho de que se trata de trabajadores que atienden al público en ventanilla . Debe llamarse la atención sobre el hecho de que esto implica un fallo o bien en el sistema de clasificación de la CNO o bien en la atribución de casos a esta categoría, ya que la clasificación incluye una categoría específica para administrativos que trabajan en ventanilla y atienden al público. Debe suponerse, además, que la distribución interna entre los administrativos entre los que atienden al público y los que trabajan con información varía con el tiempo en función de cambios organizativos en las empresas y en la administración.

Otra categoría de la CNO a tres dígitos que merece reseñarse es la de dependientes y vendedores en tiendas [533]. En este caso, una cuarta parte de los encuestados afirma trabajar en primer lugar con materiales, mientras que la mayoría lo hace con personas, como por otra parte sería previsible. Asimismo, 
entre los gestores de diverso tipo [152 y 122] y los supervisores de áreas y departamentos especializados [113] las tareas consideradas dominantes son las de trato a personas, aunque en este último grupo alrededor del $40 \%$ de los encuestados afirma que su tarea principal es el trabajo con información.

En términos generales, se pueden extraer una serie de conclusiones relevantes del examen de estos datos. En primer lugar, la población ocupada no se reparte a partes iguales entre los que trabajan prioritariamente con objetos, con datos o con personas. De hecho, no hay muchos trabajadores que afirmen trabajar con datos, y éstos están concentrados en los trabajos de administración y en los trabajos profesionales. En cualquier caso, dentro de las categorías ocupacionales hay diversidad de tareas, y esto se debe a varias razones. Una muy importante es que en ocasiones la ocupación se confunde con la profesión. Además, los supervisores tienden a responder en función de la profesión en la que se definen, y no en función de la realización de tareas de supervisión. Como resultado de todo esto, la agregación de ocupaciones en categorías que incluyen a grupos ocupacionales diferentes supone naturalmente la suma de la diversidad interna de las distintas ocupaciones, de modo tal que los tipos de tarea son internamente más diversos en las categorías agrupadas en clases más amplias que entre las categorías ocupacionales especificadas en un nivel más bajo.

\section{LA COMPLEJIDAD DE LAS TAREAS}

El resultado de la aplicación de nuestras imputaciones de niveles de complejidad a las tareas contenidas en la ocupación permite un examen más delicado de los contenidos en tareas de las categorías ocupacionales. El cuadro 7 muestra las puntuaciones de cada una de las categorías ocupacionales de la CPO para los tres tipos de complejidad, junto a su suma lineal y una suma ponderada que asigna un punto a la dificultad manual, dos a la complejidad informacional de las tareas y tres a la complejidad de trato personal. Junto al promedio de puntuación para cada categoría ocupacional se indica su desviación típica. Al pie de cada una de las columnas se indica el estadístico F para los datos presentados, la significatividad (que es menor de 0,001 en todos los casos) y la proporción de la varianza de la complejidad de tarea explicada por la clasificación de ocupaciones (o contenida en ésta) ${ }^{6}$.

Hay tres tipos de información interesante en este cuadro. Por una parte, las puntuaciones de determinadas categorías ocupacionales nos permiten una somera comprobación de la validez de nuestra medición de la complejidad o de la propia clasificación -aunque no podamos distinguir la fuente de las incongruencias si no es por medio de un ejercicio de interpretación ajeno al procedimiento operativo de medición-. En el caso de las tareas manuales, por ejemplo, encontramos un

\footnotetext{
${ }^{6}$ Este es el estadístico eta cuadrado, equivalente a la $\mathrm{R}$ cuadrado de la regresión en cuanto a su interpretación.
}

EMPIRIA. Revista de Metodología de Ciencias Sociales. N. ${ }^{\circ}$ 17, enero-junio, 2009, pp. 91-121. ISSN: 1139-5737 


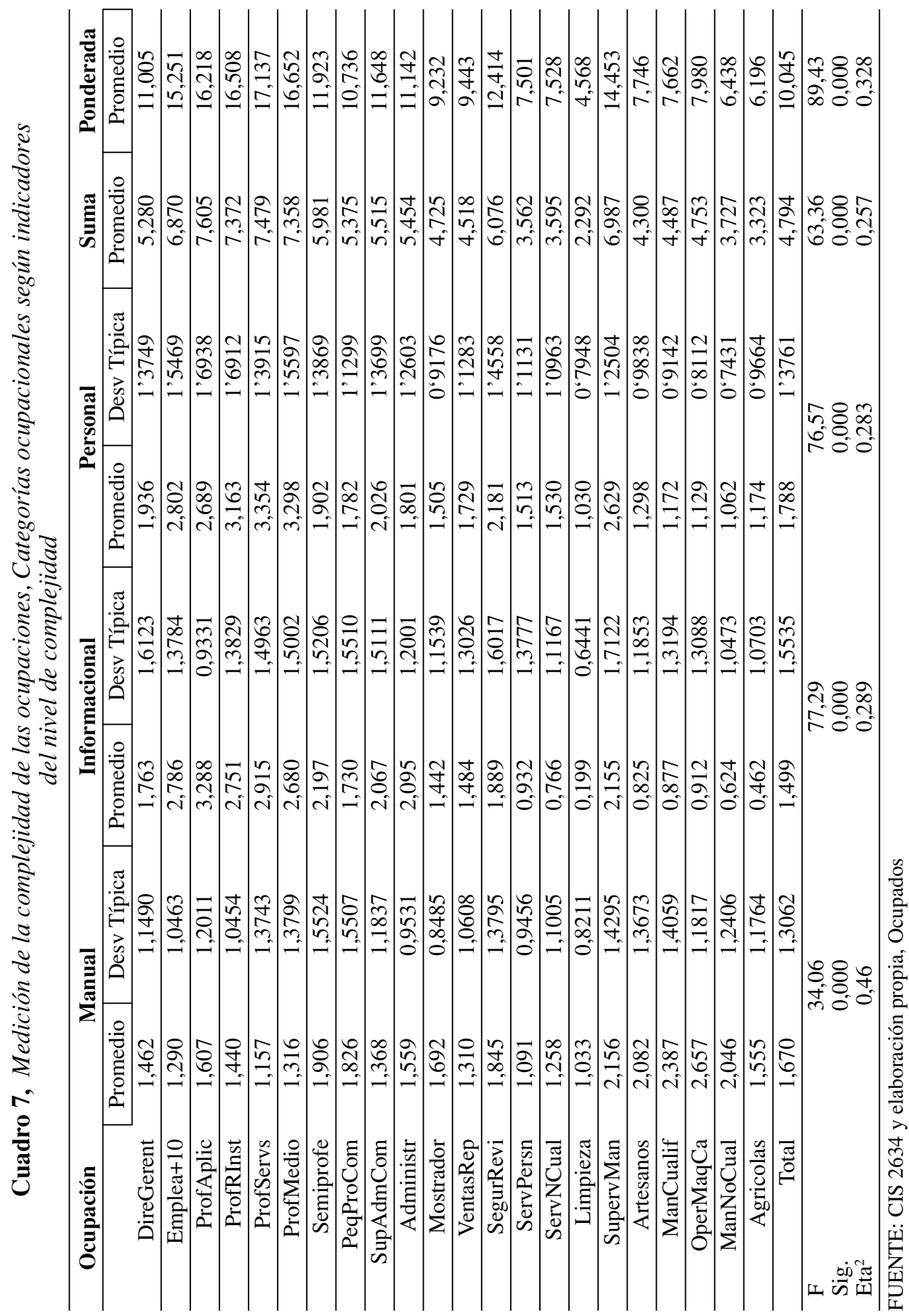

EMPIRIA. Revista de Metodología de Ciencias Sociales. N. ${ }^{\circ}$ 17, enero-junio, 2009, pp. 91-121. ISSN: $1139-5737$ 
nivel de complejidad de tarea más elevado entre las ocupaciones clasificadas como de operación de maquinaria que entre trabajadores manuales cualificados, supervisores y, sobre todo, artesanos. Eso se debe en parte a la elevada puntuación que se ha asignado al manejo de maquinaria móvil, y a la conducción de medios de transporte. Es posible, en cualquier caso, que las ocupaciones más claramente definibles como artesanales incorporen conjuntos de tareas en las que predomina la rutina mecánica sobre la evaluación de situaciones físicas.

Por otro lado, las puntuaciones en su conjunto nos permiten comprobar la coherencia de cada uno de los indicadores de complejidad, de su suma simple y de su suma ponderada. Del examen del conjunto de la tabla podemos extraer la conclusión clara de que los indicadores de complejidad están efectivamente midiendo la complejidad interna de las tareas, a juzgar por los resultados de cada una de las categorías. En ninguna de ellas se encuentra una contradicción específica entre lo que cada uno de los indicadores medidos señala que es la distribución de la complejidad de las tareas ocupacionales y lo que se podría esperar a partir de la información disponible sobre cada una de ellas. El panorama general es perfectamente coherente con las distribuciones previsibles: el trabajo manual se concentra en las ocupaciones manuales, mientras el trabajo con información se concentra en las profesionales. Las puntuaciones más elevadas en el trato con personas se encuentran en general en las ocupaciones orientadas a la supervisión técnica en el puesto (supervisores manuales y administrativos) o a la supervisión social (seguridad y revisión); pero, sobre todo, se encuentra entre los profesionales.

En tercer lugar, podemos comprobar el sentido relativo que tiene la asignación de niveles de ponderación a cada una de las medidas de complejidad. En principio, la complejidad medida como la suma de cada uno de los componentes muestra puntuaciones más elevadas en los lugares en los que se podría esperar y puntuaciones más bajas en las ocupaciones con exigencia de cualificación más humilde. Sin embargo, la métrica de la suma ponderada de las tres puntuaciones resume mejor este tipo de diferencias al aumentar su rango. Obsérvese que las diferencias presentadas entre los profesionales no son significativas según un test de Bonferroni ${ }^{7}$, como tampoco lo son para estos datos las existentes entre los trabajadores manuales. No obstante, debemos considerar válidos estos resultados en la medida en la que responden a lo que cabría esperar en una muestra más voluminosa. En conjunto, los niveles de ponderación exclusivos para cada uno de los tres tipos de complejidad (con valores 1, 2 y 3) aumentan la capacidad de predicción de la ocupación en un $7 \%$, mientras que para una ponderación que igualara el valor de tareas informacionales y personales -no mostrada en el cuadro- lo haría en un $6 \%$.

\footnotetext{
${ }^{7}$ El test de Bonferroni permite comprobar, en el contexto de un análisis de varianza, si las diferencias entre las medias de la variable dependiente en cada una de las categorías de la variable independiente son significativas. Se aplica a comparaciones por parejas entre categorías de la variable independiente, y utiliza un test t para cada una de las parejas de categorías, pero corrige la tasa de error global para que sea igual a la tasa de cada contraste dividida entre el número de contrastes.
}

EMPIRIA. Revista de Metodología de Ciencias Sociales. N. ${ }^{\circ}$ 17, enero-junio, 2009, pp. 91-121. ISSN: 1139-5737 
Cuadro 8. Complejidad de tarea de la ocupación según nivel de estudios

\begin{tabular}{|c|c|c|c|c|c|}
\hline \multirow[t]{2}{*}{ Estudios } & \multicolumn{2}{|c|}{ Suma } & \multicolumn{2}{|c|}{ Ponderada } & \multirow[t]{2}{*}{ Casos } \\
\hline & media & Desv. Tip. & media & Desv. Tip. & \\
\hline Incompletos & 2,963 & 1,957 & 5,491 & 3,753 & 108 \\
\hline Primaria & 3,412 & 1,993 & 6,307 & 3,831 & 437 \\
\hline Bach. Elemental/ESO & 3,984 & 2,369 & 7,508 & 4,778 & 1,034 \\
\hline FP Básica & 4,462 & 2,535 & 8,688 & 5,329 & 394 \\
\hline Bachillerato superior & 5,006 & 2,640 & 10,052 & 5,615 & 463 \\
\hline FP Superior & 5,476 & 2,620 & 10,586 & 5,640 & 420 \\
\hline Diplomaturas & 6,392 & 2,913 & 13,892 & 6,729 & 434 \\
\hline Superiores & 7,176 & 2,796 & 15,853 & 6,520 & 580 \\
\hline $\mathrm{F}$ & $142,84^{*}$ & $201,16^{*}$ & & & \\
\hline $\mathrm{Eta}^{2}$ & 0,206 & 0,267 & & & \\
\hline
\end{tabular}

*Significativo al $99 \%$

FUENTE: CIS 2634 y elaboración propia. Ocupados.

En relación con el problema de la conexión entre complejidad de tarea y cualificación, una aproximación básica consiste en examinar los indicadores de complejidad según el grado más alto logrado en el sistema educativo. En el cuadro 8 se muestran las diferencias en las puntuaciones de niveles de complejidad para siete categorías de niveles de estudios, reagrupadas en función de su relevancia relativa en el mercado de trabajo. Las diferencias entre las distintas categorías son en general significativas según el test de Bonferroni, con la excepción de la diferencia entre estudios incompletos y primaria y la existente entre Bachillerato y FP superior. En conjunto, los niveles de estudios explican un $26,7 \%$ de la diversidad en los niveles de complejidad de las ocupaciones.

\section{DISCUSIÓN}

Las ocupaciones se pueden analizar en términos de los objetos sobre los que se aplican las tareas que contienen, en función de la complejidad de esas tareas y en función de la cualificación requerida para lidiar con esa complejidad. Estos aspectos no agotan las posibilidades de análisis de la estructura ocupacional (Hadden, Kravets y Muntaner, 2004), puesto que otros elementos diversos forman parte también de esa variedad, especialmente relacionados con el contexto económico y social en el que tiene lugar la actividad laboral, y con el nivel de autoridad y autonomía con el que se desarrolla. Un análisis ya clásico es el realizado por Michael Hout (1984) en términos de status, autonomía y cualificación. En muchos casos, resulta difícil distinguir los aspectos más estrictamente técnicos de la estructura ocupacional -los que se abordan en esta investigación- de los contextuales: el medio organizativo en el que se desempeña la ocupación (Baron, 
Davis-Blake y Bielby, 1986), el sexo de los trabajadores (Reskin y Roos, 1990; Jacobs, 1989) y el origen étnico. En este trabajo nos hemos propuesto explorar las consecuencias de la aplicación de un estrategia analítica al estudio de la estructura ocupacional, basada en la evaluación de los propios trabajadores sobre el objeto y la complejidad de las tareas que realizan.

Los principales resultados empíricos nos muestran el potencial y la debilidad de una estrategia de este tipo. Cuando examinamos el objeto de la actividad de trabajo tal y como es evaluado por los propios trabajadores encontramos un panorama algo ambivalente. A grandes rasgos, los resultados son los que se podrían razonablemente esperar de un ejercicio de imputación técnica realizado por una entidad objetiva y externa. Sin embargo, cuando analizamos en detalle categorías ocupacionales específicas, encontramos algunas contradicciones y algunos efectos que es necesario examinar.

El origen de esos problemas se encuentra en dos aspectos del procedimiento de análisis. El primero es que estamos dando por supuesto que la evaluación realizada por los entrevistados es adecuada para un examen del objeto de las ocupaciones. Esto puede ser problemático si se acepta de un modo acrítico, puesto que las contradicciones pueden ser resultado de que los entrevistados estén equivocados o no entiendan la pregunta, de que no cuenten con la información pertinente o de que por el contrario tengan información con la que no cuenta el analista. Se puede dar el caso de que determinadas culturas profesionales supongan una definición en cuanto al objeto al que va dirigida su actividad. El segundo aspecto consiste en el hecho de que la mayor parte de las ocupaciones contienen, en su paquete de tareas, una combinación de actividades relacionadas con personas, con objetos o con información. Esto quiere decir que en unos casos es difícil evaluar cual es el objeto concreto de la ocupación, y en otros casos no hay un objeto único.

Esto se puede resolver con una estrategia del tipo de la presentada en el segundo ejercicio realizado en este artículo, consistente en la asignación a cada ocupación de un nivel de complejidad en la realización de tareas de cada uno de los tres tipos de objetos. Se reconoce de este modo el hecho de que las tareas contenidas en una ocupación están ligadas tanto a cosas, como a personas, como a objetos. De nuevo en este caso los resultados son razonablemente coherentes, pero existen algunas salvedades que deben ser discutidas. Nos encontramos con que el nivel de complejidad medido depende de los niveles previamente definidos por el analista, y esa gradación en niveles de las distintas tareas que se realizan en una ocupación no siempre es clara. Además, otra vez debemos fiarnos del criterio de los encuestados en lo referido a la dificultad de su trabajo. Sin embargo, este procedimiento tiene la ventaja de ofrecernos una fuente alternativa en el análisis de las ocupaciones, que es triangulable con los resultados derivados del análisis de la distribución ocupacional.

En último término, los problemas relacionados con la complejidad de las ocupaciones nos remiten a su conexión con la cualificación. El lector podría extraer la conclusión de que, dada la conexión entre la complejidad de los conte-

EMPIRIA. Revista de Metodología de Ciencias Sociales. N. ${ }^{\circ}$ 17, enero-junio, 2009, pp. 91-121. ISSN: 1139-5737 
nidos ocupacionales y la cualificación, el ejercicio presentado en este artículo refuerza precisamente la práctica que quiere criticar. Es decir, dado que los resultados son coherentes tanto con la distribución de las ocupaciones medida mediante una clasificación convencional, como con los niveles de estudios ¿por qué preocuparse de la complejidad y no utilizar los procedimientos convencionales? O dicho de otro modo, ¿no es la complejidad otro nombre de la cualificación?

Hay varias razones para dar una respuesta negativa (Ingram y Neumann, 2006). En primer lugar, el uso de la cualificación es tan sólo una aproximación al valor real de la complejidad de tarea, dado el hecho de sobra conocido de que no todo el mundo tiene una ocupación acorde a su cualificación. La complejidad es una cosa distinta de la cualificación. En realidad, en ésta se mezclan dos componentes: uno relacionado con la titularidad formal de capacidades de trabajo (credenciales) y otro relacionado con la adquisición de habilidades. La cualificación, en cualquier caso, es una propiedad del trabajador, mientras la complejidad lo es del puesto de trabajo. Mientras el ajuste de la complejidad al cambio técnico es inmediato, el de la cualificación es lento y está sometido a redefiniciones sociales. Este es el aspecto más problemático del uso de la cualificación como elemento diferenciador en las clasificaciones de ocupaciones.

En segundo lugar, la complejidad de tarea no es un sustituto para muchos de los usos de las clasificaciones de ocupaciones. En el análisis de las consecuencias sociales de la estructura ocupacional la importancia de ésta está relacionada con el hecho de estar ligada a oportunidades o condiciones de vida y trabajo diversas, y no de un modo directo con la complejidad de las tareas. El interés de analizar la complejidad intrínseca del desempeño de los empleos se encuentra precisamente en que ésta tiene sus propias consecuencias: en términos de cambios en los contenidos de la estructura ocupacional, o en términos de transformaciones en los requisitos de cualificación en el mercado de trabajo y en el valor de mercado de las cualificaciones.

Una estrategia de investigación en el futuro debería pasar por el estudio de las relaciones internas entre los distintos componentes de la complejidad y otros aspectos de las ocupaciones. Las relaciones de autoridad en el empleo presentan un reto particularmente complicado, puesto que sabemos que están mal medidas por las clasificaciones estándar. Una segunda línea de análisis potencial nos debe llevar a refinar los procedimientos de medición de la complejidad de las tareas, que como se ha mostrado a lo largo de este trabajo no son sencillos. El problema principal, a mi juicio, es decidir cual es la ordenación correcta de las tareas según su complejidad, y generar la capacidad de realizar una medida cuantitativa del nivel de complejidad que no suponga, como se ha hecho aquí, una imputación por parte del investigador. Una tercera línea debe llevarnos, en fin, a analizar las posibles consecuencias sociales de los cambios en la complejidad interna de las ocupaciones, no solamente en términos de cambios en las exigencias de cualificación, sino también en la propia definición de las ocupaciones.

EMPIRIA. Revista de Metodología de Ciencias Sociales. N. ${ }^{\circ}$ 17, enero-junio, 2009, pp. 91-121. ISSN: 1139-5737 


\section{BIBLIOGRAFÍA}

ABBOT, Andrew (1993): «The Sociology of Work and Occupations». En Annual Review of Sociology, Vol. 19, pp. 187-209.

ANDERSEN, Erling B. (1997): Introduction to the Statistical Analysis of Categorical Data. Heidelberg, Springer.

BARON, James N., Alison DAVIS-BLAKE y William T. BIELBY (1986): «The Structure of Opportunity: How Promotion Ladders Vary Within and Among Organizations». En Administrative Science Quarterly, Vol. 31, n. 1, pp. 248-273.

BENIGER, James R. (1990): «Conceptualizing Information Technology as Organization, and Viceversa». En Fulk, Janet y Charles W. Steinfield (1990) Organizations and Information Technology. Sage, Newbury Park.

CARABAÑA, Julio, y Carmuca GÓMEZ BUENO (1994): Una escala cardinal de prestigio profesional. Documento de trabajo 94-01. Madrid, IESA-CSIC.

CARABAÑA, Julio, y Carmuca GÓMEZ BUENO (1996): Escalas de prestigio profesional. Madrid, CIS.

COLLADO, Juan Carlos, e Isabel MARTINEZ (1995): «Nivel de estudios y ocupaciones en la estructura sectorial del empleo. Análisis comparativo con la UE». En Economistas, $\mathrm{n}^{\mathrm{0}}$ 69, pp. 336-342.

COXON, Anthony P.M. y Charles L. JONES (1979): Classes and Hierarchy. The Social Meaning of Occupations. Londres, MacMillan.

DESROSIERES, Alain, y Laurent THEVENOT (1990) : «Les Categories Socio-Professionelles», en Revue francaise de sociologie, vol. 31, nํ 3, pp. 505-507.

ETZIONI, Amitai (1969) The Semi-Professions and their Organization: Teachers, Nurses and Social Workers. Nueva York/Londres, The Free Press.

GARRIDO, Luis (1991): Prospectiva de las ocupaciones y de la formación en la España de los 90. Madrid, Ministerio de Economía y hacienda.

GONZÁLEZ, Juan Jesús (1992a): «La construcción empírica de las clases», en Política y Sociedad, no 11, pp. 99-122.

GONZÁLEZ, Juan Jesús (1992b): Clases sociales: estudio comparativo de España y la Comunidad de Madrid, 1991. Madrid, Servicio estadístico de la Comunidad Autónoma de Madrid.

GREEN, Francis (1999): The Market Value of Generic Skills. Skills Task Force Research Paper, Londres.

HADDEN, Wilbur, Nataliya KRAVETS y Carles MUNTANER (2004): «Descriptive Dimensions of US Occupations with data from O*NET». En Social Science Research, Vol. 33, n. 1, pp. 64-78.

HOUT, Michael (1984): «Status, Autonomy and Training in Occupational Mobility». En American Journal of Sociology, Vol. 89, n. 6, pp. 1379-1409.

INGRAM, Beth F. y George R. NEUMANN (2006): «The Returns to Skill». En Labour Economics, Vol. 13, n. 1, 35-59.

JACOBS, Jerry (1989) Revolving Doors. Sex Seggregation and Women's Careers. Stanford University Press, Stanford.

JONES, Frank E. (1980): «Skill as a Dimension of Occupational Classification», en $\mathrm{Ca}$ nadian Review of Sociology and Antrhopology, vol. 17, no 2, pp. 176-183.

NEILSEN, David (1986): «Towards a Framework for Socioeconomic Classification». En New-Zealand Sociology, vol.1, no 1, pp. 34-49. 
O'REILLY, Karen, y David ROSE (1998): Conceptualizing Social Divisions: The ESRC Review of UK Government social Classifications. Documento de trabajo de la ASA.

OPITZ, Otto, Berthold LAUSEN y Rudiger KLAR (1993): Information and Classification. Concepts, Methods and Applications. Springer, Berlin.

PIERCE, John R. (1961l1980): An Introduction to Information Theory. Simbols, Signals and Noise. Nueva York, Dover.

POPPING, Roel (1995): «The Performance of a Knowledge-Based System for Converting Occupational Classifications». En Quality and Quantity, no 29, pp. 273-286.

RESKIN, Barbara y Patricia ROOS (Eds.) (1990): Job Queues, Gender Queues. Women's Inroads into Male Occupations. Temple University Press, Philadelphia.

RIVIÈRE, Jaime (2004): «Olas de recualificación: Estructura ocupacional, organizaciones y educación en España». Presentado en el VIII Congreso Español de Sociología, Alicante, 23 al 25 de septiembre de 2004. Publicado como Documento de Trabajo del Departamento de Sociología y Comunicación de la Universidad de Salamanca en 2007.

SCOTT, Richard W. (1981/2003): Organizations. Rational, Natural and Open Systems. Prentice Hall, Upper Saddle River.

THEVENOT, Laurent (1983) : «A propos d'une definition de couches moyennes et de la nouvelle nomenclature des professions et categories», en Revue francaise de Sociologie, vol. 24, no 2, pp. 317-326.

USDL (1991): Dictionary of Occupational Titles. 4th Edition. United States Department of Labor, Washington DC.

WEAVER, Warren (1948): «Science and Complexity». En American Scientist, Vol. 36,, pg. 536.

WIRTH, Ulrich (1993): «Caminalcules and Didaktozoa: Imaginary Organisms and Textexamples for Systematics». En Opitz, Lausen y Klar (1993).

WOLF, Christoph (1997): «The ISCO-88 International Standard Classifiction of Occupations in Cross-National Research». En Bulletin de Methodologie Sociologique, $\mathrm{n}^{\mathrm{o}}$ 54 , pp. 23-40.

ZHOU, Xueguang (1992) : «Occupational Classifications as Institutions». ASA working papers, Ithaca. 


\section{ANEXO}

Construcción de la clasificación de ocupaciones utilizada a partir de la CNO94

\begin{tabular}{|c|c|c|c|}
\hline Categoría & Código & Contenido y ejemplos & $\begin{array}{l}\text { Correspondencia } \\
\text { con CNO94 }\end{array}$ \\
\hline Directivos y gerentes & DirGerent & $\begin{array}{l}\text { Directivos y gerentes de } \\
\text { administraciones públicas } \\
\text { y empresas }\end{array}$ & 10 A 17 \\
\hline Empleadores +10 & Emplea+10 & $\begin{array}{l}\text { Empleadores de empresas } \\
\text { de más de } 10 \text { trabajadores }\end{array}$ & 11, empleadores \\
\hline Profesionales aplicados & ProfAplic & $\begin{array}{l}\text { Ingenieros, matemáticos, } \\
\text { físicos, arquitectos, } \\
\text { químicos }\end{array}$ & 20 \\
\hline $\begin{array}{l}\text { Profesionales de regulación } \\
\text { Institucional }\end{array}$ & ProfRInst & $\begin{array}{l}\text { Profesionales del derecho, } \\
\text { de la economía, de las } \\
\text { ciencias sociales y de la } \\
\text { organización de empresas }\end{array}$ & 23,24 \\
\hline $\begin{array}{l}\text { Profesionales servicios } \\
\text { sociales }\end{array}$ & ProfServs & $\begin{array}{l}\text { Médicos, psicólogos, } \\
\text { periodistas, profesionales } \\
\text { de las ciencias naturales, } \\
\text { enseñantes }\end{array}$ & $21,22,25$ \\
\hline Profesionales medios & ProfMedio & $\begin{array}{l}\text { Profesiones asociadas a un } \\
\text { primer ciclo universitario }\end{array}$ & 26 A 29 \\
\hline Semiprofesionales & Semiprofe & $\begin{array}{l}\text { Técnicos en ciencias y } \\
\text { sanidad, educación infantil, } \\
\text { vuelo, sistemas de video y } \\
\text { audio }\end{array}$ & 30 A 32,35 \\
\hline $\begin{array}{l}\text { Pequeños Propietarios del } \\
\text { comercio y de los servicios }\end{array}$ & PeqProCom & $\begin{array}{l}\text { Pequeños propietarios no } \\
\text { profesionales ni agrarios }\end{array}$ & $\begin{array}{l}\text { Distinto de } 11 \text { a } 18 \text {, } \\
\text { no prof. } \\
\text { Empleadores }\end{array}$ \\
\hline Administrativos & Administr & $\begin{array}{l}\text { Servicios contables, } \\
\text { financieros, de producción } \\
\text { y transporte, bibliotecas, } \\
\text { auxiliares administr. }\end{array}$ & $34,40 \mathrm{~A} 43$ \\
\hline Empleados de mostrador & Mostrador & $\begin{array}{l}\text { Administrativos con tareas } \\
\text { de atención al público, } \\
\text { recepcionistas, telefonistas, } \\
\text { cajeros }\end{array}$ & 44 A 46 \\
\hline Ventas y representación & VentasRep & $\begin{array}{l}\text { Agentes de seguros, de } \\
\text { viaje, inmobiliarios, } \\
\text { tasadores, repr. de } \\
\text { comercio, dependientes, } \\
\text { ventas }\end{array}$ & 33,53 \\
\hline
\end{tabular}

EMPIRIA. Revista de Metodología de Ciencias Sociales. N. ${ }^{\circ}$ 17, enero-junio, 2009, pp. 91-121. ISSN: 1139-5737 


\begin{tabular}{|c|c|c|c|}
\hline Categoría & Código & Contenido y ejemplos & $\begin{array}{l}\text { Correspondencia } \\
\text { con } \mathrm{CNO94}\end{array}$ \\
\hline Seguridad y Revisión & SegurRevi & $\begin{array}{l}\text { Militares, policías, prof. de } \\
\text { la seguridad, bomberos, } \\
\text { guardas jurados }\end{array}$ & 00,52 \\
\hline Servicios Personales & ServPersn & $\begin{array}{l}\text { Cocineros y camareros, } \\
\text { bármanes, azafatas, cuidado } \\
\text { de personas, peluqueros }\end{array}$ & 50,51 \\
\hline Servicios no cualificados & ServNCual & $\begin{array}{l}\text { Vendedores a domicilio, } \\
\text { conserjes, vigilantes, } \\
\text { ordenanzas, mozos, lect. de } \\
\text { contadores, basureros }\end{array}$ & $90,92,93$ \\
\hline Limpieza & Limpieza & $\begin{array}{l}\text { Empleadas del hogar y } \\
\text { personal de limpieza de } \\
\text { hoteles y oficinas }\end{array}$ & 91 \\
\hline Supervisores manuales & SupervMan & $\begin{array}{l}\text { Encargados de obra, } \\
\text { capataces, supervisores de } \\
\text { talleres mecánicos e inst. } \\
\text { industriales }\end{array}$ & $70,73,80,82$ \\
\hline Artesanos & Artesanos & $\begin{array}{l}\text { Mec. de precisión, artes } \\
\text { gráficas, ceramistas, } \\
\text { artesanos de madera, textil } \\
\text { y cuero, ebanistas, } \\
\text { matarifes }\end{array}$ & $77,78,79$ \\
\hline Manual Cualificado & ManCualif & $\begin{array}{l}\text { Pescadores, ferrallistas, } \\
\text { pintores, mineros, } \\
\text { soldadores, herreros, } \\
\text { chapistas, ajust. de } \\
\text { maquinaria }\end{array}$ & $63,71,72,74$ A 76 \\
\hline Operarios de maquinaria & OperMaqCa & $\begin{array}{l}\text { Operadores de instal. Ind., } \\
\text { de maq. Fijas, montadores, } \\
\text { maquinistas, conductores }\end{array}$ & 81,83 A 86 \\
\hline $\begin{array}{l}\text { Trabajadores no } \\
\text { cualificados }\end{array}$ & ManNoCual & Peones de la industria & 95 A 98 \\
\hline $\begin{array}{l}\text { Trabajadores de la } \\
\text { agircultura, propietarios, } \\
\text { obreros y jornaleros }\end{array}$ & Agricolas & $\begin{array}{l}\text { Agricultores y trabajadores } \\
\text { cualificados en actividades } \\
\text { agrícolas y ganaderas } \\
\text { Peones agropecuarios }\end{array}$ & 60 А 62,94 \\
\hline
\end{tabular}

Elaboración propia

EMPIRIA. Revista de Metodología de Ciencias Sociales. N. ${ }^{\circ}$ 17, enero-junio, 2009, pp. 91-121. ISSN: 1139-5737 


\title{
RESUMEN
}

Las ocupaciones tienen al menos tres componentes claramente diferenciables: los relacionados con su dificultad como actividad, los relacionados con su contenido jerárquico y los relacionados con el objeto al que se aplican los procesos de trabajo. En este estudio se lleva a cabo un análisis de los contenidos de las ocupaciones en relación con el objeto y la complejidad de las tareas en los distintos grupos ocupacionales. En primer lugar, se discuten las dificultades de la medición de la complejidad de las tareas contenidas en las ocupaciones. Después se examinan las orientaciones de las ocupaciones hacia tipos de objetos de trabajo específicos. Por último, se muestra la posibilidad real de medir la complejidad de las tareas en el puesto de trabajo y se discuten las consecuencias de este ejercicio de medición. Para la realización de estos análisis se utiliza el estudio 2634 del CIS sobre «estratificación social».

\section{PALABRAS CLAVE}

Trabajo, estratificación, ocupaciones, complejidad, clasificación

\begin{abstract}
Occupations have three distinct elements: those related with their difficulty as activity, those related with their hierarchical dimension and those related with the object to wich the work process is applied. This study analyzes occupational contents in relation with the complexity and object of different occupational groups. First, obstacles in the measurement of task complexity are discussed. Then we inspect the orientation of occupations towards specific objects of work. Finally, the real chance of task complexity measuring is shown, with a discussion of the consequences of this measurement exercise. This analysis is performed with the CIS survey 2634 on «Social Stratification».
\end{abstract}

\section{KEYWORDS}

Work, stratification, occupations, complexity, classification 